\title{
Multiscale Experiments and Modeling in Biomaterials and Biological Materials, Part II
}

\author{
JING DU (1), ${ }^{1,5}$ DINESH KATTI, ${ }^{2,3,6}$ and HENDRIK HEINZ ${ }^{4,7}$ \\ 1.-Department of Mechanical Engineering, Pennsylvania State University, \\ University Park, PA 16802, USA. 2.-Center for Engineered Cancer Testbeds, North Dakota \\ State University, Fargo, ND, USA. 3.-Department of Civil and Environmental \\ Engineering, North Dakota State University, Fargo, ND, USA. 4.-Department of Chemical \\ and Biological Engineering, University of Colorado Boulder, Boulder, CO, USA. \\ 5.—e-mail: jingdu@psu.edu. 6.-e-mail: dinesh.katti@ndsu.edu. 7.—e-mail: hendrik.heinz@ \\ colorado.edu
}

The first part of this special topic on multiscale experiments and modeling in biomaterials and biological materials was published in June 2021. The second part, presented here, features a series of papers on natural biological materials, including molecules, cells, and tissues. Also featured is a collection of papers on human-made biomaterials, linking their macroscopic behaviors to the underlying microstructures. Experimentally, the assessment of macroscopic mechanical properties and the characterization of microscopic structures are presented. Additionally, computational methods from the atomic level to macroscale are applied, including the latest artificial intelligence algorithms.

Cellulose is a natural biopolymer that can be found in plant cell walls such as algae, vegetables, wood, and cotton and can be used in paper and clothing textiles. Sun and his team presented their multiscale computational work on cellulose in the paper "A new parameterization of all-atom force field for cellulose." Quantum mechanics (QM) density functional theory (DFT) was applied to obtain the parameters for the all-atom force field. Molecular dynamics (MD) simulations were carried out at different temperatures and compared with experimental data to obtain the temperature-dependent parameters.

CoVE is the smallest structural membrane protein of the SARS-CoV-2 virus. A paper titled "Selforganized morphology and multi-scale structures of CoVE proteins" was contributed by Sompornpisut and Pandey. In this work, coarse-grained Monte Carlo simulations were performed to calculate the

(Received May 29, 2021; accepted June 2, 2021;

published online June 21, 2021) self-organizing structures of CoVE proteins at various temperatures and with various protein densities.

The advent of high-throughput microscopy in both clinical and research environments for imaging of biological cells necessitates the fast and accurate identification and analysis of a large number of cells of different types. Kalidindi and coworkers' paper, "Automated image processing workflow for morphological analysis of fluorescence microscopy cell images," describes a new automated workflow for automated analysis of cellular data. The new workflow is far more accurate at identifying cell images from various backgrounds mimicking real-world microscopy images. The authors have made the framework available to researchers via an opensource platform.

Predicting the elastic modulus of bone based on clinical images is of great clinical importance but is difficult because the bone is a complex hierarchical porous material. Wang and his group contributed a paper titled "Prediction of elastic behavior of human trabecular bone using a DXA image-based deep learning model." They simulated the elastic deformation of trabecular bone specimens using finite element modeling based on micro X-ray computed tomography (micro-CT). Then the convolutional neural network, a deep learning model, was trained and tested using dual-energy x-ray absorptiometry (DXA) images of trabecular bone specimens and the apparent elastic moduli determined from finite element simulations. The results show that, compared with the conventional regression model, the deep learning model gave a more accurate prediction of the elastic behaviors of human trabecular bone. 
Hip replacement by implants made of artificial materials is a necessity for many patients and improves function and quality of life. These surgeries have become relatively common. The formation of the scar tissue around the implants is important for joint stability. In the paper "A homogenized macro scale model and a morphological micro scale model to understand the varying mechanical properties of scar tissues of hip capsule ligaments grown around different implant materials," the study led by Budyn and coworkers evaluated the mechanical properties of scar tissue at various length scales resulting from implants made of polymer, metal, and ceramic and compared them with properties of native tissue obtained during initial surgeries. This foundational work could lead to the development of a protocol for the selection of implant material based on patient-specific parameters.

Bioinspired materials and structures are a field of great interest and promise to design revolutionary materials and structures with unprecedented properties. In the paper titled "Jaws of Platynereis dumerilii: miniature biogenic structures with hardness properties similar to those of crystalline metals," Hellmich and coworkers have explored the structure-property relationship in the microscopic jaw structures of the cosmopolitan bristle worm Platynereis dumerilii. Through nanoindentation combined with detailed chemical and structural analysis, the authors have revealed key features that seem to control the extraordinary mechanical properties of the jaw structures. The metal-like biogenic materials in the jaw structures, with the secrets of the origin of the properties expounded by the authors, could lead to groundbreaking new biomimetic structural materials.

The paper by Li and coworkers, "Evaluation and modeling of processability of laser removal technique for bamboo outer layer," deals with developing processability parameters and modeling of a laser removal technique used for stripping the outer layer of bamboo, an important construction material. The authors investigated several crucial parameters such as removal depth, surface roughness, and color under various radiation modes and modeled them. As a result, the paper provides optimized laser parameters for effectively stripping the outer layer of bamboo.

Colloidal particles are the nanoscopic building blocks in the design of hydrogels, which can be used in drug delivery systems, biomolecular sensors, tissue engineering, and scaffolds for cell growth. In the paper "Design of functionalized lobed particles for porous self-assemblies," Vashisth and his group used coarse-grained Langevin dynamics simulations to calculate the morphologies self-assembled by functionalized lobed particles. The selfassembly behaviors were studied for lobed particles with various shapes, such as snowman, dumbbell, trigonal planar, tetrahedral, square planar, trigonal bipyramidal, and octahedral.

Carvalho et al., contributed a paper, "Evaluation of $\mathrm{MgZnCa}$ alloys fabricated via powder metallurgy for manufacturing biodegradable surgical implants." In this work, two MgZnCa alloys, Mg$5 \mathrm{Zn}-1 \mathrm{Ca}$ and $\mathrm{Mg}-29 \mathrm{Zn}-2 \mathrm{Ca}$, were fabricated by powder metallurgy. Their mechanical properties, corrosion resistance, microstructure, and degradation rate were characterized. The results show that the mechanical resistance, hydrogenous dissolution, and corrosion reduction of $\mathrm{Mg}-29 \mathrm{Zn}-2 \mathrm{Ca}$ were comparable with other as-cast $\mathrm{Mg}$ alloys. Its degradation rate was found to be similar to other $\mathrm{Mg}$-based alloys. Hence, it can potentially be used for biodegradable implants.

Calcium phosphates are bioceramics commonly used for orthopedic implants because of their excellent bioactivity and bone-bonding ability. Unlike other calcium phosphates, such as hydroxyapatite, fluorapatite (Fap) is less studied. Guidara et al. contributed a paper titled "Structural and mechanical properties of porous $\mathrm{Al}_{2} \mathrm{O}_{3}-\mathrm{Fap}-\mathrm{TiO}_{2}$ composite as a promising material for bone implants." Porous $\mathrm{Al}_{2} \mathrm{O}_{3}-\mathrm{Fap}-\mathrm{TiO}_{2}$ composite ceramics were fabricated with a porosity of $50 \%$ to $53 \%$ and interconnected pores ranging between 43 and $280 \mu \mathrm{m}$. The compressive strength of the composites was measured to be $14 \mathrm{MPa}$ to $18 \mathrm{MPa}$, which is higher than those of the human cancellous bone. The results indicate the porous $\mathrm{Al}_{2} \mathrm{O}_{3}-\mathrm{Fap}-\mathrm{TiO}_{2}$ composite is a promising material for bone implants.

Hydrogels and ferrogels are cross-linked polymeric networks with three-dimensional structures, having applications in joint prosthesis, catalyst, wound healing, biosensing, contact lenses, and drug delivery. In the paper, "A review on material's design and biomedical applications of hydrogels and ferrogels," Awasthi introduced the fabrication method, structures, (mechanical, tribological, and magnetic) properties, and applications of hydrogels/ferrogels. Their interactions with cells and metallic, carbon, and silicon nanoparticles are also included. The potential applications of hydrogels/ferrogels in biomedical engineering are discussed.

Polymer-ceramic composite materials have vast applications in the biomedical field as a substitute for native biological tissues, specifically those with structural/mechanical functions. Du and coworkers have studied the crack propagation in one such material, epoxy-alumina composite, which they had previously investigated for dental applications and contributed a paper titled "Investigation of internal cracks in epoxy-alumina using in situ mechanical testing coupled with micro-CT." The clever use of in situ mechanical testing coupled with micro-CT experiments provides rare insight into the fracture mechanisms in the important composite system. The authors elucidate the toughening mechanisms 
and suggest ways to improve the fracture toughness of the material to be effectively and safely used for biomedical applications.

The authors Tsige and coworkers studied the effect of hydration and intermolecular interactions on adhesion in mussel-inspired soybean-based adhesive. The mussel-inspired adhesives that use "foot proteins" show strong underwater adhesion. The authors in their paper, "Single chain hydration and dynamics of mussel-inspired soybean-based adhesive," describe the effect of hydration on adsorption on substrates, an important consideration for the design of high-performance adhesives.

To read or download any of the papers from this topic, follow the URL http://link.springer.com/journ $\mathrm{al} / 11837 / 73 / 8$ to the table of contents page for the August 2021 issue (vol. 73, no. 8). To see the June issue papers in this topic, go to http://link.springer.c om/journal/11837/73/6.

\section{ACKNOWLEDGEMENTS}

J.D. acknowledges funding by the National Science Foundation under Grant Numbers CMMI1826221. D.K. acknowledges funding from National Science Foundation Grant Number OIA- \#1946202.

\section{DECLARATIONS}

\section{CONFLICT OF INTEREST}

The authors declare that they have no conflict of interest.

Publisher's Note Springer Nature remains neutral with regard to jurisdictional claims in published maps and institutional affiliations. 